with 22 gauge FNA needle with no on site pathologist and direct expulsion of material into cyto-rich red medium. A standard 3 passes were performed in all lesions. Patient were excluded if they did not have a gold standard comparison for FNA results which was defined as surgical specimen comparison, death resulting from disease or clinical follow up and imaging for a minimum of 6 months.

Results 831 EUS procedure were performed during the study period of which 129 had FNA of a solid pancreatic lesions. 23 patents had a SEMS in situ at the time of EUS FNA and 5 had a plastic stent. The accuracy of pancreatic FNA with a SEMS was $65 \%$ $(15 / 23)(95 \% \mathrm{CI}$ : $44-81 \%)$ a plastic stent was $80 \%(4 / 5)(95 \% \mathrm{CI}$ : $36 \%$ to $98 \%)$ and with no stent was $84.3 \%(86 / 102)(95 \%$ CI:76$90 \%)$ and overall was $82.2 \%(106 / 129)(95 \%$ CI: $75-88 \%)$. The FNA accuracy for the presence of a metallic stent was significantly lower $\chi^{2}=4.4(p=0.036)$. All 8 patients with a SEMS had a false negative result of which 5 were felt well enough to undergo a further procedure for consideration of chemotherapy which gave an accuracy of $80 \%(4 / 5)$. All patients were happy to undergo a repeat biopsy.

Conclusion The accuracy for FNA of solid pancreatic lesions in the presence of a SEMS can be significantly lower than without and should be taken into account when consenting patients and planning treatment. However it should not delay the insertion of a SEMS as if definitive cytology is required a repeat FNA is a feasible option with an acceptable accuracy.

Disclosure of Interest None Declared

\section{OC-043 THE ROLE OF THE EPS8 BINDING PARTNERS SOS1 AND ABI1 IN PANCREATIC CANCER}

doi:10.1136/gutjnl-2013-304907.042

1."P Kiely, ' J Tod, 'V Jenei, 'C Johnson, 'G J Thomas. 'Cancer Sciences, University of Southampton School of Medicine, Southampton, UK

Introduction Pancreatic cancer (PC) is an aggressive disease, characterised by marked local invasion, and identification of new molecular targets is critical to improving outcome. Cell motility requires reorganisation of the actin cytoskeleton and several actin-binding proteins have been implicated in PC. EGF receptor pathway substrate 8 (Eps8) is an adapter protein that interacts with a range of intracellular binding partners, including Abi1 and Sos1 (to form a tri-complex), and certain $\beta$ integrin subunits, in order to regulate cytoskeletal reorganisation. The integrin $\alpha v \beta 6$ is overexpressed in approximately $70 \%$ of PC and enhances invasion. This study examines the role of the Eps8 binding partners, Abi1 and Sos1, in $\alpha v \beta 6$-dependent PC invasion and the significance of their expression on patient survival.

Methods We used immunochemistry to examine the expression of Eps8, Sos1 and Abi1 in normal pancreas and PC in vivo using tissue microarrays. A retrospective patient database of PC patients operated on between 2000 and 2010 was generated. 33 short ( $<1$ year) and 20 long ( $>5$ years) survivors were then identified and resection tissue stained as whole sections for Eps8/ Sos1/Abi1/ $\alpha v \beta 6$. We identified three PC cell lines that showed $\alpha v \beta 6$-dependent invasion in vitro, and examined the role of Abi1 and Sos1 in Transwell ${ }^{\circledR}$ assays to specifically study motility dependent on this integrin.

Results Eps8, Sos1 and Abi1 were up-regulated in PC compared with normal tissue. Expression of these proteins in long and short survivors of PC is currently being examined. Expression of Eps8, Sos1, Abi1 and $\alpha v \beta 6$ were confirmed in all three PC cell lines tested. Knock-down of Eps8, Sos1 or Abi1 suppressed $\alpha v \beta 6$ dependent invasion suggesting that this tri-complex is critical to PC motility.
Conclusion We have shown that Eps8, Sos1 and Abi1 are upregulated in PC and regulate $\alpha v \beta 6$-dependent function. The Eps 8 binding partner Sos1 appears to be critical to $\alpha v \beta 6$-dependent PC cell motility. This may be of particular interest as Sos 1 expression was previously shown to fall in response to gemcitabine, the current gold standard chemotherapeutic agent for the treatment of PC. Sos1 requires further investigation as a potential molecular target in the treatment of PC.

Disclosure of Interest None Declared

\section{Gastrointestinal physiology associates (AGIP) symposium}

\section{OC-044 EFFECTS OF SLEEVE GASTRECTOMY ON GASTRO- OESOPHAGEAL REFLUX AND OESOPHAGO-GASTRIC MOTILITY}

doi:10.1136/gutjnl-2013-304907.043

1."J 0 Hayat, ${ }^{2 S}$ Mansour, ${ }^{2} \mathrm{~A}$ Wan, ${ }^{1} \mathrm{~A}$ P Poullis, ${ }^{3} \mathrm{E}$ Yazaki, ${ }^{3} \mathrm{D}$ Sifrim, ${ }^{1} \mathrm{~J}-\mathrm{Y}$ Kang. ${ }^{1}$ Gastroenterology; ${ }^{2}$ Bariatric Surgery, St.George's University of London; ${ }^{3}$ Wingate Institute of Neurogastroenterology, Barts and the London School of Medicine and Dentistry, London, UK

Introduction Sleeve gastrectomy is increasingly used as both a definitive and staggered weight loss procedure. The effect of sleeve gastrectomy on gastro-oesophageal reflux (GOR) remains uncertain. We studied the effect of sleeve gastrectomy on GOR, pressure parameters at the Lower Oesophageal Sphincter (LOS), oesophageal and gastric motility.

Methods Sixteen patients (median age 46 (range 25-71)) with morbid obesity underwent high resolution oesophageal manometry and $24 \mathrm{hr}$ ambulatory $\mathrm{pH}$-impedance monitoring, at least 2 weeks pre-op and 3 months post sleeve gastrectomy (median no. of days post-op 129 (range 84-202)). All patients documented reflux and dysphagia symptoms at the time of testing. Nine patients also underwent concurrent gastric emptying with ${ }^{13} \mathrm{C}$ labelled octanoate breath test. Parametric data was analysed using the paired t-test and non-parametric data with Wilcoxon matched pairs test.

Results Mean Body Mass Index (BMI) fell from 49 (41.3-58.3) to 38.5(35-46.3). 5/16 patients reported new or worsening reflux symptoms (31\%). Basal LOS pressure fell from $14.4 \mathrm{mmHg}(9.7-28.5)$ preop to $8.9 \mathrm{mmHg}(0.7-40.5)$ post-op $(\mathrm{p}<0.02)$. Intra-gastric pressure and G-O pressure gradient increased: $8.3 \mathrm{mmHg}(4.7-12.8)$ pre-op Vs $10.4 \mathrm{mmHg}(5.3-22.7)$ post-op $(\mathrm{p}<0.01)$. 8/16 patients had severe hypomotility pre-op and $9 / 16$ post-op. $3 / 16$ patients had pathological acid reflux pre-op, $5 / 16$ patients having de novo reflux post-op. Mean total acid exposure time pre-op was $1.8 \%(0.5-5 \%)$ increasing to $4.35 \%(0.2-12.4 \%)(p<0.02)$ post-op. There was an increase in the number of acid: $18(8-31)$ pre-op vs $29(13-38)$ post-op $(p<0.0001)$, and non-acid reflux episodes: 13(7-19) pre-op vs 52(35-84) post-op(p $<0.0001)$. Non-acid reflux episodes occurred predominantly in the post-prandial period. Gastric half emptying time(t1/2) was significantly shorter post-op 193.1mins (range113-433) vs $115.8 \mathrm{mins}$ (range 82-170) ( $p<0.05)$

Conclusion Both acid and non-acid gastro-oesophageal reflux is increased after sleeve gastrectomy with $31 \%$ of patients developing de novo acid reflux post-op. This is despite a reduction in BMI and accelerated gastric emptying. A reduction in LOS pressure and increased G-O pressure gradient are likely to be contributing factors. Future studies should determine whether decreased gastric compliance stimulates increased numbers of transient lower oesophageal sphincter relaxations.

Disclosure of Interest None Declared 